\title{
Non-radioactive detection of dot-blotted genomic DNA for species identification
}

\author{
TR Chen, C Dorotinsky \\ American Type Culture Collection, 12301 Parklawn Drive, Rockville, MD 20852, USA \\ (Proceedings of the 9th European Colloquium on Cytogenetics of Domestic Animals; \\ Toulouse-Auzeville, 10-13 July 1990)
}

species identification / biotinylation / genomic DNA

\section{INTRODUCTION}

The purpose of this study was to find a simple DNA-based procedure for species identification to be used for quality control in cell culture systems. Isoenzymology and cytogenetics are frequently used for identifying species origin of a cell line. However, these methods are labor intensive and technically demanding and results are sometimes indefinite. Additional simple, sensitive and reproducible methods to identify species would be a welcome progress. Herein, we report a procedure to identify the genomic DNA (gDNA) of an unknown species, dot-blotted on a nitrocellulose filter, by hybridizing with biotinylated, non-radioactive DNA probes from a known species. By selecting the appropriate probes, this procedure can be used to distinguish species gDNAs clearly.

\section{MATERIALS AND METHODS}

\section{Materials}

Cell lines and plasmid DNAs containing different chromosome segments used for this study are listed in tables I and II. They are all deposited at the ATCC.

\section{Procedures}

DNA extraction. The standard phenol-chloroform method was used for DNA extraction and purification (Maniatis et al, 1982). The amount of DNA was measured by spectrophotometry.

Biotinylation. Both biotin-14-dATP (BRL) and biotin-21-dUTP (BioRad) were used. Methods to biotinylate DNA probes followed manufacturers' recommendations with slight modifications as follows: 1) $1.5-2.0 \mu \mathrm{g}$ of the DNA probe was used per reaction; 2) reaction time was extended to $5-7 \mathrm{~h}$ before being stopped with $0.3 \mathrm{M}$ ethylenediaminetetraacetic acid (EDTA); and 3 ) the reaction solution was used directly for hybridization. 
Table I. List of species origin of cell lines used for this study.

\begin{tabular}{|c|c|c|c|c|c|c|c|c|c|c|}
\hline \multirow[t]{2}{*}{ Cell line } & \multicolumn{3}{|c|}{ Cell line identification } & \multirow[t]{2}{*}{ Content } & \multicolumn{6}{|c|}{ Reaction intensity ${ }^{a}$ of probes } \\
\hline & $A T C C$ no & $s p p$ & $\begin{array}{c}\text { common } \\
\text { name }\end{array}$ & & $H s a$ & $M m u$ & Rno & $H / M$ & $8 q$ & $13 q$ \\
\hline HeLa & CCL-2 & $H s a$ & human & genomic & 5 & 0 & 0 & 2 & 5 & 5 \\
\hline NCI-H716 & CCL-251 & $H s a$ & human & genomic & 5 & 0 & 0 & 2 & 5 & 5 \\
\hline SK-UT-1A & НTB-114 & $H s a$ & human & genomic & 5 & 0 & 0 & 2 & 5 & 5 \\
\hline SK-UT-1B & HTB-115 & $H s a$ & human & genomic & 5 & 0 & 0 & 2 & 5 & 5 \\
\hline Tera-1 & HTB-105 & $H s a$ & human & genomic & 5 & 0 & 0 & 2 & 5 & 5 \\
\hline Carl & CRL-1857 & Ptr & chimpanzee & genomic & 5 & 0 & 0 & & & \\
\hline Rock & CRL-1854 & Ggo & gorilla & genomic & 5 & 0 & 0 & & & \\
\hline Puti & CRL-1850 & $P p y$ & orangutan & genomic & 5 & 0 & 0 & & & \\
\hline CV-1 & CCL-70 & Cae & $\begin{array}{l}\text { African green } \\
\text { monkey }\end{array}$ & genomic & 5 & 0 & 0 & & & \\
\hline $1283 \mathrm{Lu}$ & CRL-6297 & $S n i$ & marmoset & genomic & 5 & 0 & 0 & & & \\
\hline $\mathrm{LM}(\mathrm{tk})$ & CCL-1.3 & $M m u$ & mouse & genomic & 0 & 5 & 2 & 5 & 0 & 0 \\
\hline $\mathrm{H}_{4} \mathrm{TG}$ & CRL-1578 & Rno & rat & genomic & 0 & 2 & 5 & & & \\
\hline $\mathrm{CHO}-\mathrm{K} 1$ & CCL-61 & $C g r$ & Ch hamster & genomic & 0 & 2 & 2 & & & \\
\hline $\mathrm{CAOV}-3$ & HTB-75 & $?$ & $?$ & genomic & 5 & 0 & 0 & & & \\
\hline H99 & & $H / M$ & hybrid & $t(13 q 17 q)^{1}$ & 2 & 5 & 2 & 5 & & \\
\hline $\mathrm{H} 101$ & & $H / M$ & hybrid & $\mathrm{N} 17(\mathrm{H})$ & 1 & 5 & 2 & 5 & & \\
\hline
\end{tabular}

a Arbitrary scale in ascending order of reaction intensity from 0 to 5 where 0 is a negative reaction (or no hybridization) and 5 is the strongest positive reaction

Table II. List of plasmids with specific human chromosome DNA segments.

\begin{tabular}{|c|c|c|c|c|c|c|c|c|}
\hline \multirow[t]{2}{*}{ Clone } & \multicolumn{2}{|c|}{ Probe identification } & \multicolumn{6}{|c|}{ Reaction intensity ${ }^{\mathrm{a}}$} \\
\hline & probe locus & map & Hsa & $P t r$ & Ggo & Ppy & Cae & $S n i$ \\
\hline TL11 & D8S5 & $8 \mathrm{q} 22-\mathrm{q} 23$ & 5 & 5 & 5 & 5 & 5 & 5 \\
\hline cMHZ47 & D13S52 & $13 q(\mathrm{H})$ & 5 & 5 & 5 & 5 & 5 & 5 \\
\hline pE25.a & $\mathrm{D} 1 \mathrm{Z7}$ & $\mathrm{N} 1 \alpha$ & 5 & 4 & 5 & 3 & 0 & 0 \\
\hline pJM128 & D8Z2 & $\mathrm{N} 8 \alpha$ & 5 & 2 & 2 & 5 & 0 & 0 \\
\hline pA10RR8 & D10Z1 & $\mathrm{N} 10 \alpha$ & 5 & 4 & 4 & 3 & 0 & 0 \\
\hline $\mathrm{pA} 12 \mathrm{H} 8$ & Do0Z0 & $\mathrm{N} 12 \alpha$ & 5 & 4 & 4 & 4 & 0 & 0 \\
\hline $\mathrm{L} 1.26$ & D13Z1 & $\mathrm{N} 13 \alpha$ & 5 & 3 & 4 & 5 & 0 & 0 \\
\hline L1.84 & D18Z1 & $\mathrm{N} 18 \alpha$ & 5 & 2 & 2 & 5 & 0 & 0 \\
\hline \multirow[t]{2}{*}{ p22hom 48.4} & $\mathrm{D} 22 \mathrm{Z3}$ & N22non- $\alpha$ & 5 & 3 & 2 & 5 & 0 & 0 \\
\hline & & & $45^{\mathrm{b}}$ & 32 & 33 & 40 & 10 & 10 \\
\hline
\end{tabular}

a Arbitrary scale in ascending order of reaction intensity from 0 to 5 where 0 is a negative reaction (or no hybridization) and 5 is the strongest positive reaction.

b Accumulated values of the reaction intensity. They may be used to assess the phylogenetic relationship between species. See the text for a detailed explanation. 
Blotting. Following the standard method (Maniatis et al, 1982), denatured gDNAs were blotted onto the nitrocellulose membrane filters using a minifold apparatus (Schleicher \& Schuell, Inc). The blotted membrane was incubated in the vacuum oven for $2-3 \mathrm{~h}$ at $80^{\circ} \mathrm{C}$.

Hybridization. $15-30 \mu \mathrm{l}$ of biotinylated probe were used for each reaction that used a $70 \mathrm{~cm}^{2}$ filter and $3.5 \mathrm{ml}$ of prehybridization or hybridization solution supplemented with an appropriate amount of freshly denatured salmon sperm DNA (Maniatis et al, 1982). Prehybridization and hybridization incubations were, respectively, $3 \mathrm{~h}$ and overnight at $42^{\circ} \mathrm{C}$.

Detection. Biotinylated DNA was detected with streptoavidin-alkaline phosphatase conjugate according to the methods recommended by the manufacturer.

\section{RESULTS}

Qualitative and quantitative differences in hybridization to the gDNA on the nitrocellulose membranes were clearly demonstrated with non-radioactive biotinylated DNA probes. Positive hybridization was revealed by the streptoavidin-alkaline phosphatase conjugate detection system. When monitored properly, $50 \mathrm{ng}$ of gDNA produced, within $5 \mathrm{~min}$, the distinct deep blue color of the maximum +5 scale adopted for our evaluation. This method is simple, reproducible and uncompromising.

Tables I and II summarize hybridization results. Probes using gDNA from human (Hsa), mouse (Mmu) and rat (Rno) cell lines showed a lack of hybridization between primates and rodents (fig 1). By contrast, weak to moderate cross-reactions were observed among three rodent species, Mmu, Rno and Chinese (Ch) hamster (Cgr) (fig $1 \mathrm{~b}, \mathrm{c}$ ). Thus, gDNA can be used as the DNA probe to distinguish distantly related species.

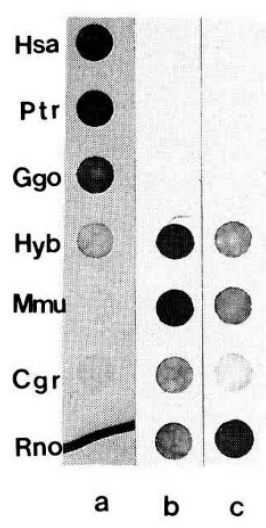

Fig 1. A panel of gDNAs blotted in order, from top to bottom: Hsa, Ptr, Ggo, Hsa/Mmu, (Hyb), Mmu, Cgr and Rno. Biotinylated gDNA probes from Hsa (a), Mmu (b) and Rno (c) were used for hybridization. Note the weak reactions in the hybrid, Hyb (a), Cgr and Rno (b), and Hyb, Mmu and Cgr (c). See text for further details. 
Within primates, human gDNA showed substantial cross-hybridization with gDNAs from chimpanzee (Ptr), gorilla (Ggo), orangutan (Ppy), African green monkey (Cae) and marmoset (Sni) (eg, fig 1a). When DNAs from human chromosome N8 or an N13 segment were used as the probe, they cross-reacted with all primate species studied here (fig $2 \mathrm{a}, \mathrm{b}$ ), producing a result similar to that of the gDNA. These data demonstrate the substantial homology in these species.

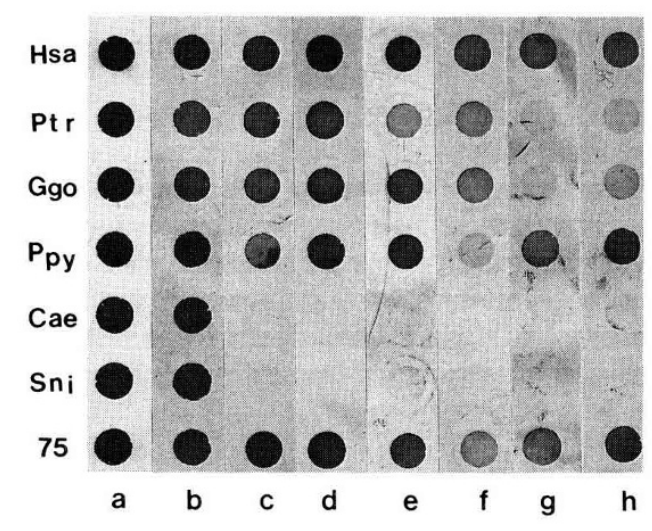

Fig 2. A panel of gDNAs blotted in order, from top to bottom: Hsa, Ptr, Ggo, Ppy, Cae, Sni and the indicator, 75 . They were hybribized with biotinylated gDNA probes from Hsa (a), a segment from human chromosome N13q (b), N10q (c) and N12q (d); non-alphoid sDNA from N22 (e); alphoid sDNA from N1 (f), N8 (g) and N18 (h).

Alphoid satellite DNAs (sDNA) from human chromosomes N10 and N12 distinguished African green monkey and marmoset from the remaining 4 species (fig 2c, d). The non-alphoid sDNA from human N22 suggested a difference between chimpanzee and the Hsa-Ggo-Ppy species (fig 2e). Alphoid sDNA from human N1 reacted similarly to N10 and N12, except that with the orangutan gDNA which appeared relatively weak (fig 2f). Alphoid sDNA from N8 and N18, however, clearly distinguished not only the Cae-Sni from the other 4 species, but also the Hsa-Ppy from the Ptr-Ggo (fig $2 \mathrm{~g}, \mathrm{~h}$ ). Experiments to further discriminate between these probes for species identification are currently in progress.

Probe DNA from an N13q segment identified the man-mouse cell hybrid H99 but not the hybrid H101 line, which, respectively, possessed only human $t(13 q 17 q)$ or N17 (fig 3). The potential use of this procedure for syntenic gene-chromosome mapping by means of somatic cell hybridization is proposed.

\section{DISCUSSION AND CONCLUSION}

Since the isolation of genomic DNA is a simple, routine procedure, and the reagents for biotinylation and detection are available commercially in kit form, this nonradioactive biotinylation procedure can be performed by most laboratories. No cross-hybridization was detected using rodent or human gDNAs. Mouse and rat gDNAs showed quantitative distinctions between 3 rodent species. Thus, these 


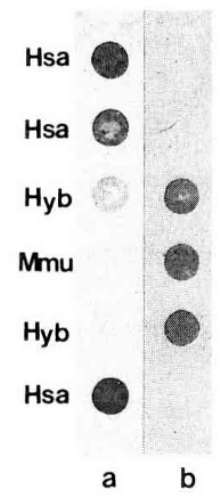

Fig 3. A panel of gDNAs blotted in order, from top to bottom: Hsa-1, Hsa-2, H99 (Hyb), Mmu, H101 (Hyb), and Hsa-3. They were hybridized with biotinylated probes from a human N13q segment (a) and gDNA from Mmu (b).

gDNAs readily distinguish distantly related species. Since most cell lines are derived from these 4 species, this procedure is a useful means for quality control of cell cultures. Moreover, plasmid DNA containing a chromosome segment can be used to identify the presence of that chromosome in a hybrid cell line genome; it provides a simple alternative for the identification of chromosomes and is also useful for gene mapping studies by somatic cell hybridization.

Specific DNA probes can be used to identify phylogenetically close species. DNA from some human chromosomes or chromosome segments cross-reacted with 6 primate species. However, alphoid DNAs from different human chromosomes are more specific, since they show differential cross-reactions. The molecular aspects of the complexity of these alphoid sDNAs have been the subject of many studies (see reviews by Singer, 1982; Willard and Waye, 1987). Cytogenetically, this complexity is also apparent, since individual chromosome identification requires a step for chromosome in situ suppression (CISS) to further eliminate the non-specific oligoDNA repeats (Lichter et al, 1990). As also demonstrated herein, alphoid sDNA probes from different human chromosomes recognized species-specific properties among the 6 primate species tested. Thus, this DNA subfamily can be used not only to identify individual chromosomes (Pinkel et al, 1986; Lichter et al, 1988, 1990), but also to distinguish between closely related species. Moreover, it may give inferences as to the phylogenetic relationship between species, as the data on N8 and N18 alphoid DNAs suggest a closer affinity between human and orangutan than between human and chimpanzee-gorilla.

\section{REFERENCES}

Lichter P, Cremer T, Borden J, Ward DC (1988) Delineation of individual human chromosomes in metaphase and interphase cells by in situ suppression hybridization using recombinant DNA libraries. Hum Genet 80, 224-234 
Lichter P, Chang-Tang CJ, Call K, Hermanson G, Evans GA, Housman D, Ward DC (1990) High-resolution mapping of human chromosome 11 by in situ hybridization with cosmid clones. Science 247, 64-69

Maniatis T, Fritsch EF, Sambrook J (1982) Molecular Cloning: A Laboratory Manual. Cold Spring Harbor Laboratory, Cold Spring Harbor, NY

Pinkel D, Straume T, Gray JW (1986) Cytogenetic analysis using quantitative, high sensitivity, fluorescence hybridization. Proc Natl Acad Sci USA 83, 2934-2938

Singer MF (1982) Highly repeated sequences in mammalian genomes. Int Rev Cytol 76, $67-112$

Willard HF, Waye S (1990) Hierarchial order in chromosome-specific human alpha satellite DNA. Trend Genet 3, 192-198 